\title{
Producción de alevinos en Orestias luteus (carachi amarillo) mediante reproducción artificial con alimentación natural para su conservación en el lago Titicaca, Puno-Perú
}

Production of alevinos in Orestias leteus (Carachi Amarillo) by artificial reproduction with natural feeding for conservation in lake Titicaca, Puno-Peru

Recibido: mayo 11 de 2016 | Revisado: agosto 10 de 2016 | Aceptado: setiembre 15 de 2016

Juan Mamani Ochochoque ${ }^{\mathrm{I}}$ ShedA MÉndez ANCCA ${ }^{2}$

1 Institución Educativa Privada "SIGMA". Puno, Perú juanorestias@hotmail.com

2 Universidad Nacional de Moquegua. Moquegua, Perú shedamendez@gmail.com

\section{Resumen}

La extinción de especies nativas del lago Titicaca resulta uno de los mayores problemas no solo para la salud humana por consumo alimentario, sino además en la subsistencia propia de las especies. El objetivo de esta investigación fue producir alevinos en la especie Orestias luteus mediante reproducción artificial con alimentación natural para su conservación en el lago Titicaca, Puno-Perú. El estudio se realizó durante los meses de febrero hasta noviembre del 2013. Para la conservación, se analizó la fecundación artificial mediante el método seco, así como la producción de cadena alimentaria viva en ambiente controlado. La alimentación consistió en microalgas y microcrustáceos. Las variables controladas fueron la temperatura, $\mathrm{pH}$ y oxígeno disuelto. Se obtuvo un porcentaje de fecundación artificial un $92 \%$ de ovas fecundadas, $96 \%$ de larvas como de alevinos, donde la alimentación con microcrustáceos representó la más significativa. Se concluyó que la producción de alevinos en la especie Orestias luteus fue alta lo cual indica que puede permitirse el procedimiento realizado para su conservación en el lago Titicaca.

Palabras clave: alevinos, alimentación natural, conservación, lago Titicaca, producción, Orestias luteus, Puno

\section{Abstract}

The extinction of native species of Lake Titicaca is one of the biggest problems not only for human health due to food consumption, but also in the subsistence of species. The objective of this research was to produce fingerlings of the species Orestias luteus through artificial reproduction with natural feeding to be preserved in Lake Titicaca, Puno-Peru. The study was carried out during from February to November of 2013. For the conservation, the artificial fertilization was analyzed using the dry method, as well as the production of live food chain in a controlled environment. The food consisted of microalgae and microcrustaceans. The variables controlled were temperature, $\mathrm{pH}$ and dissolved oxygen. We achieved a $92 \%$ of artificial fertilization of fertilized eggs, $96 \%$ of fingelings offspring, where feeding with microcrustaceans 
represented the most significant. It was concluded that the production of fingerlings in the species Orestias luteus was highly significant, which indicates that the procedure performed for its conservation in Lake Titicaca can be afforded.

Key words: fingerlings, natural feeding, conservation, Lake Titicaca, production, Orestias luteus, Puno.

\section{Introducción}

Actualmente, la contaminación de los ecosistemas acuáticos lénticos representa uno de los principales problemas ambientales por resolver (Samantray, Mishra, Panda $\&$ Rout, 2009), debido a que afecta la biodiversidad endémica, donde tal situación en sinergia con otras variables ambientales como las meteorológicas, pueden ocasionar efectos adversos como por ejemplo, la mortandad masiva de peces en el lago Titicaca dado los efectos de remoción de ecotoxicidad local (Argota, 2015).

El lago Titicaca es el más grande de agua dulce en América del Sur con una superficie de $8300 \mathrm{~km}^{2}$, longitud máxima de 195 $\mathrm{km}, 285$ metros de profundidad máxima y un ancho media de $50 \mathrm{~km}$, siendo el lago navegable más alto del mundo $(3808 \mathrm{~m} \mathrm{~s}$. $\mathrm{n}$. m. ). Tanto el sector peruano como boliviano están incluidos en la lista de humedales de importancia internacional después de la Convención de Humedales Ramsar, 1971, donde presenta una biodiversidad tanto en flora como fauna acuática (Constantini, Savetta, Mancinelli \& Rossi, 2004).

Este lago Titicaca simboliza para la comunidad local, un medio de desarrollo debido a la piscicultura intensiva que se realiza entre otras actividades. Sin embargo, Buschmann (2001) refiere que la piscicultura intensiva puede tener un impacto negativo sobre el ecosistema, debido a la acumulación de materia orgánica generada sobre los fondos procedentes de las excretas, materia orgánica muerta y la fracción de alimento no consumido, respectivamente.

La actividad de sobrepesca, de igual forma amenaza la biodiversidad fundamentalmente de especies ícticas nativas, reconocidas popularmente como mauri, suche, ispi y carachi, donde sus poblaciones han disminuido significativamente (Mantilla, 2004). En el caso de la especie Orestias luteus (carachi amarillo), su disminución es generada precisamente por la propia sobrepesca, la cual se desarrolla, fundamentalmente, en zonas ribereñas del lago (Chura \& Mollocondo, 2009), estimándose que de las especies nativas (representan los mayores índices de capturas), el género Orestias, es el mayor por su demanda $(70,6 \%)$, seguidamente de la trucha y pejerrey $(30,0 \%)$ que han sido introducidas y finalmente, Trichomycterus $(2,5 \%)$ donde destacan tanto mauri como suche, respectivamente.

En lo publicado por el propio Argota (2015), refiere que el Laboratorio Continental de Puno reportó que durante el evento sucedido en marzo de 2013 dada la mortandad masiva de peces, se produjo una pérdida de biomasa para Orestias luteus, alrededor de $702.0 \mathrm{Kg}$. siendo $44 \mathrm{mil}$ en número de ejemplares. Ante tal situación, se hace necesaria la búsqueda de repoblamiento de forma inmediata, para mantener a la Orestias luteus dentro de la biodiversidad íctica, siendo la 
técnica de reproducción artificial, una de las mejores opciones científicas, pero la misma deberá estar condicionada a programas de adecuada alimentación.

El objetivo de esta investigación fue producir alevinos en la especie Orestias luteus mediante reproducción artificial con alimentación natural para su conservación en el lago Titicaca, Puno-Perú.

\section{Método}

\section{Objeto de investigación y periodo de es- tudio}

El estudio se realizó en la zona litoral del lago Titicaca. Para ello, fueron muestreados individuos reproductores de la especie Orestias luteus (carachi amarillo), en que los análisis se efectuaron durante los meses de febrero hasta noviembre del 2013.

\section{Población y muestra}

Para la captura de los individuos reproductores, se instalaron redes agalleras de 1,71,8 cocadas. Se tuvo en cuenta que los peces estuvieran vivos para lo cual los especímenes se extrajeron de la red directamente a un contenedor con agua. La captura o recolección de los especímenes en las redes se realizó en tempranas horas de la mańana. Luego se efectuó la extracción de ovas y espermatozoides de los reproductores aptos o grávidos.

\section{Análisis de las variables}

- Fecundación artificial mediante el método seco

Una vez sexados los individuos adultos fértiles (separación de machos y hembras), se obtuvo las ovas de las hembras (translucidas y amarillentas) y el esperma en machos (líquido blanquecino), a través del orificio urogenital. Luego se realizó el frezamiento, que es un método que consiste en fecundar los ovocitos, de forma artificial, depositando los espermas sobre las ovas y dejando reposar tres minutos para que se realice la fecundación, donde de forma anticipada se vertió gotas de agua que facilitó la movilidad de los espermas.

Es importante que durante el proceso, anteriormente, mencionado, se realice un lavado con abundante agua para eliminar determinadas impurezas. Luego se trasladan las muestras hacia un vaso chasse; se agrega, aproximadamente, dos litros de agua hasta cubrir completamente las muestras, a fin de propiciar aireación mecánica durante 24 horas generando movimiento circular constante.

Seguidamente, se procedió a la desaglutinación de los huevos 24 horas después de la fecundación, una vez que las ovas adquirieron mayor resistencia, por medio de la fricción de los dedos, lavando nuevamente las muestras para eliminar todo tipo de residuos que quedasen, evitando con ello, la contaminación de las ovas fecundadas por hongos.

Durante el conteo de las ovas, a través del método volumétrico (correspondiendo a la primera fase de incubación) se deposita, en una probeta graduada $(1 \mathrm{ml})$, hasta proceder a su conteo. Luego se realizó la incubación durante 22 días a una temperatura de $15^{\circ} \mathrm{C}$, donde diariamente, se realizó recambio de agua, extraer las ovas muertas y determinadas impurezas, donde finalmente ocurrió la eclosión, proceso que puede durar dos días y que depende de la temperatura y la higiene del agua y así obtener, en este periodo, las larvas (Figura 1) con presencia del saco vitelino. Una vez reabsorbido el saco vitelino, las larvas pasan a ser alevinos que son peces pequeños cuyas características morfológicas y la coloración aún no se parece a la etapa adulta (Figura 2). 


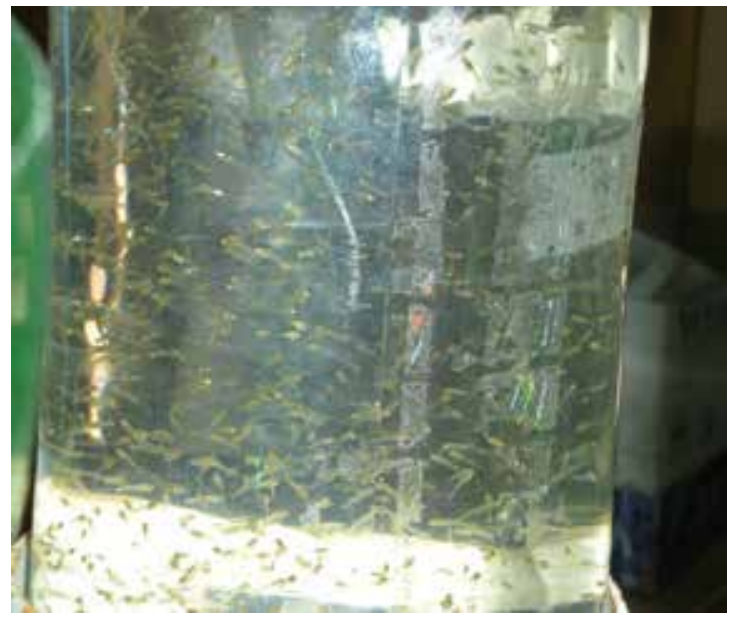

Figura 1. Presencia de larvas en el acuatorio. IMARPE, 2015

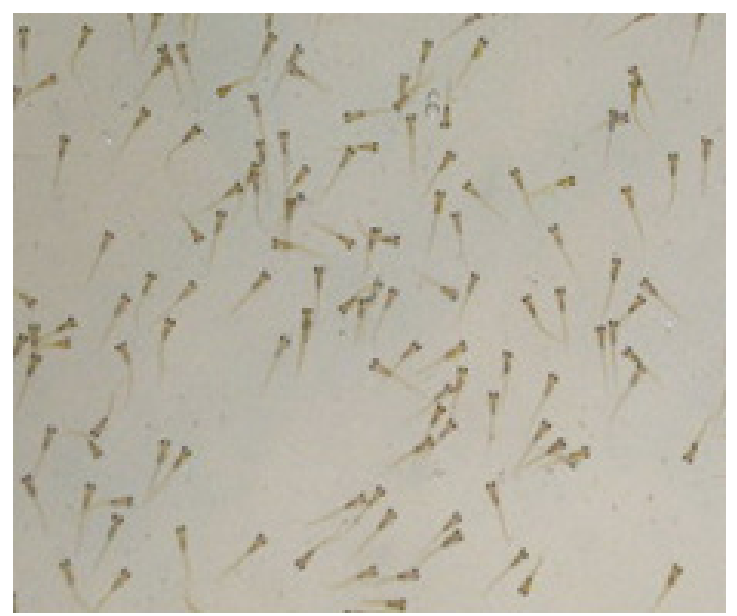

Figura 2. Presencia de alevinos en el acuatorio. IMARPE, 2015

- Control de parámetros

Los parámetros que fueron controlados correspondieron a la temperatura, $\mathrm{pH}$ y oxígeno disuelto para lo cual se utilizaron instrumentación, previamente, calibradas y certificadas. En cuanto a la alimentación, correspondió a microalgas y microcrustáceos. Las microalgas se obtuvieron del lago Titicaca mediante el método de arrastre con una red fitoplanctónica. Luego se procedió a la siembra de las microalgas en matraces para producir un litro de microalgas (Figura 3).

En el caso de los microcrustáceos, se obtuvo igualmente del lago Titicaca por medio del método de arrastre con una red fitoplanctónica, donde se procedió luego

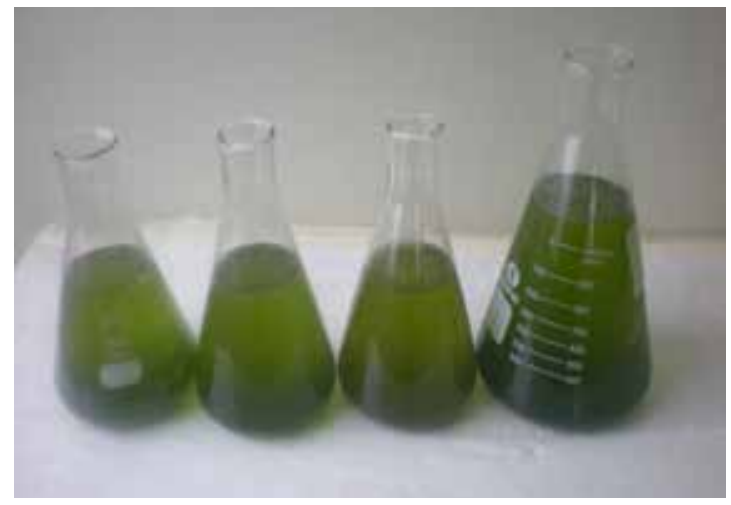

Figura 3. Presencia de microalgas. IMARPE, 2015

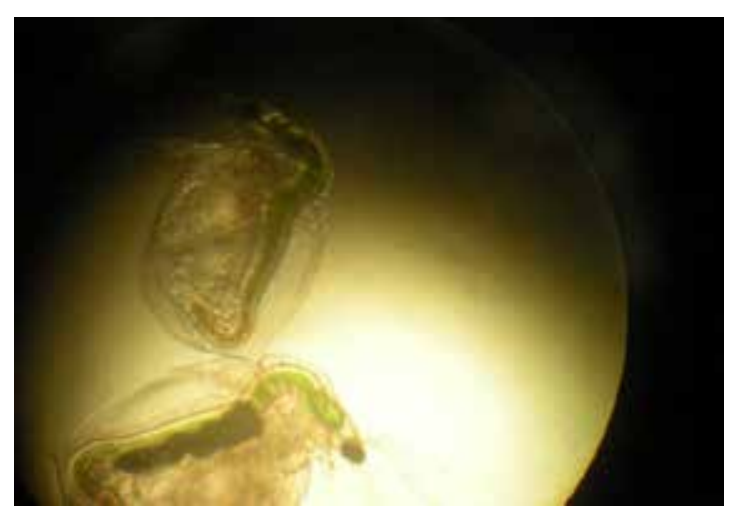

Figura 4. Presencia de microcustráceos en cámara de vidrio. IMARPE, 2015

a la siembra de los mismos en matraces para producir un litro de microcrustáceos (Figura 4).

Las condiciones de experimentación para la alimentación consistieron en la selección aleatoria de 20 alevinos, suministrándoles microalgas, microalgas-microcrustáceos y microcrustáceos (rotíferos, cladóceoros y copépodos). La alimentación no fue suministrada ad-libintum.

\section{Análisis estadístico de los datos}

Para el análisis estadístico de los datos, se utilizó el programa profesional Statgraphics versión 5.1 (copyrigth: 19942001). Se utilizó como estadígrafo descriptivo paramétrico de tendencia central el porciento, tanto para las larvas obtenidas de las ovas incubadas y en la formación de alevines durante dos meses. 


\section{Resultados}

La Tabla 1 muestra por cada tratamiento, la determinación sobre la fecundación artificial del carachi amarillo mediante el método seco.

Tabla 1

Fecundación artificial en Orestias luteus

\begin{tabular}{|c|c|c|}
\hline \multirow{2}{*}{ Tratamiento } & \multicolumn{2}{|c|}{ Muestra (1:1) } \\
\cline { 2 - 3 } & Hembras & Machos \\
\hline 1 & 26 & 26 \\
\hline 2 & 16 & 16 \\
\hline 3 & 18 & 18 \\
\hline Total & $\mathbf{6 0}$ & $\mathbf{6 0}$ \\
\hline
\end{tabular}

La Tabla 2 muestra por cada tratamiento el porcentaje de obtención de ovas fecundadas del carachi amarillo mediante el método seco.

\section{Tabla 2}

Porcentaje de ovas fecundadas en Orestias luteus

\begin{tabular}{|c|c|c|c|}
\hline $\begin{array}{c}\text { Trata- } \\
\text { mientos }\end{array}$ & $\begin{array}{c}\text { No. de } \\
\text { ovas } \\
\text { fecun- } \\
\text { dadas }\end{array}$ & $\begin{array}{c}\text { No. de } \\
\text { ovas } \\
\text { muertas } \\
(\mathbf{1 4 5} / \mathbf{m l})\end{array}$ & $\begin{array}{c}\text { No. de } \\
\text { ovas } \\
\text { vivas } \\
(\mathbf{1 4 5} / \mathbf{m l})\end{array}$ \\
\hline 1 & 6500 & 520 & 5980 \\
\hline 2 & 4800 & 375 & 4405 \\
\hline 3 & 5819 & 442 & 5377 \\
\hline Total & 17,119 & 1,337 & 15,762 \\
\hline$\%$ & $\mathbf{1 0 0 . 0 0}$ & $\mathbf{8 . 0 0}$ & $\mathbf{9 2 . 0 0}$ \\
\hline
\end{tabular}

La Tabla 3 muestra por cada tratamiento, el porcentaje de obtención de larvas fecundadas del carachi amarillo mediante el método seco.

Tabla 3

Porcentaje de larvas fecundadas en Orestias luteus

\begin{tabular}{|c|c|c|c|}
\hline $\begin{array}{c}\text { Trata- } \\
\text { mientos }\end{array}$ & $\begin{array}{c}\text { No. de } \\
\text { larvas }\end{array}$ & $\begin{array}{c}\text { No. de } \\
\text { larvas } \\
\text { muertas } \\
(\mathbf{1 4 5 / m l})\end{array}$ & $\begin{array}{c}\text { No. de } \\
\text { larvas } \\
\text { vivas } \\
(\mathbf{1 4 5} / \mathbf{m l})\end{array}$ \\
\hline 1 & 5980 & 336 & 5644 \\
\hline 2 & 4405 & 111 & 4294 \\
\hline
\end{tabular}

\begin{tabular}{|c|c|c|c|}
\hline 3 & 5377 & 109 & 5247 \\
\hline Total & 15,762 & 556 & 15,185 \\
\hline $\mathbf{\%}$ & $\mathbf{1 0 0 . 0 0}$ & $\mathbf{4 . 0 0}$ & $\mathbf{9 6 . 0 0}$ \\
\hline
\end{tabular}

La Tabla 4 muestra por cada tratamiento, el porcentaje de obtención de alevinos del carachi amarillo

\section{Tabla 4}

Porcentaje de alevinos en Orestias luteus

\begin{tabular}{|c|c|c|c|}
\hline $\begin{array}{c}\text { Trata- } \\
\text { mientos }\end{array}$ & $\begin{array}{c}\text { No. de } \\
\text { alevinos }\end{array}$ & $\begin{array}{c}\text { No. de } \\
\text { alevinos } \\
\text { muertos } \\
(\mathbf{1 4 5} / \mathbf{m l})\end{array}$ & $\begin{array}{c}\text { No. de } \\
\text { alevinos } \\
\text { vivos } \\
(\mathbf{1 4 5} / \mathbf{m l})\end{array}$ \\
\hline 1 & 5644 & 356 & 5280 \\
\hline 2 & 4294 & 108 & 4186 \\
\hline 3 & 5247 & 136 & 5111 \\
\hline Total & 15,185 & 600 & 14577 \\
\hline$\%$ & $\mathbf{1 0 0 . 0 0}$ & $\mathbf{4 . 0 0}$ & $\mathbf{9 6 . 0 0}$ \\
\hline
\end{tabular}

\section{Discusión}

El $92.00 \%$ de ovas fecundadas vivas, obtenidas de Orestias luteus es un indicador que mostró la posibilidad de aplicar la reproducción artificial mediante el método seco con excelentes resultados, siendo comparativamente significativo los resultados obtenidos Meneses (1995), quien realizó un trabajo de investigación sobre la reproducción artificial mediante el método seco en Orestias pentlandii en la laguna de Arapa, obteniendo ovas fecundadas e inclusive hasta lograr larvas en proceso de reabsorción de saco vitelino. En este estudio, se logró obtener, adicionalmente, a las larvas, la etapa de alevinaje.

Según Huet (1988) y la Organización de las Naciones Unidas para la Alimentación y la Agricultura (FAO, 2002), el método seco es el más recomendable en la fecundación artificial, a pesar del inconveniente sobre la aglutinación de los huevos que fueron separados mecánicamente, donde este causó mortalidad durante el corte de membranas en el momento de la fecundación, por cuanto se realizó dicha aglutinación 24 horas después de la fecundación, ya que en este 
momento las ovas adquirieron mayor resistencia y con ello se disminuyó la mortalidad. La aplicación del método seco ha sido utilizada, en otros estudios, como, por ejemplo, durante la evaluación de tres protocolos de tratamiento hormonal sobre el diámetro de ovocitos de sabaleta Brycon henni (Lenis, Restrepo \& Cruz, 2009), así como durante el desempeño reproductivo del bocachico Prochilodus magdalenae inducido dos veces, en un mismo ańo (Atencio, Kerguelen, Naar \& Petro, 2013), respectivamente.

Para este trabajo, se realizó un procedimiento similar al realizado por Llanos y Scotto (2014), con relación a la fecundación artificial en peces, en que la manipulación técnica durante la inseminación artificial se logró induciendo a las hembras a expulsar sus ovas, así como los espermatozoides en los machos.

Luego de realizar la producción de la cadena de alimentación viva en ambientes controlados para alevinos de Orestias luteus, con lo que se obtuvo tanto la producción de microalgas como microcrustáceos, pudo observarse que la preferencia alimentaria correspondió a los microcrustáceos y son los nauplios, la dieta durante los primeros treinta días, debido no solo a su lenta locomoción, sino además por su tamańo que posibilitan la entrada para su digestión a través de la boca. Posteriormente, la alimentación preferencial hasta los sesenta días de edad son los cladóceros y luego copépodos hasta los diez meses de edad sin que se presente anomalías morfo-fisiológicas.
Atencio (2001) indicó que es necesario realizar el seguimiento post eclosión como son la etapa de larvaje y alevinaje, en donde se debe suministrar principalmente alimentos naturales. En este estudio, se recolectó plancton propiamente del lago, garantizando el desarrollo por etapa. En el informe final del Centro de Investigación y Desarrollo Acuícola Boliviano (CIDAB, 2002), donde se aplicó reproducción artificial con Trichomycterus sp. (mauri), los resultados arrojaron que fue posible obtener larvas bajo suministrar alimentación artificial con zooplancton y fitoplancton extraídos del lago Titicaca; sin embargo, no pudo obtener alevinos.

En el presente trabajo experimental se pudo obtener alevinos de Orestias luteus en ambientes controlados, donde se evidenció que dicha especie posee un patrón alimentario carnívoro, manifestándose luego de la absorción del saco vitelino (reserva energética), etapa donde se presenta mortalidad en la especie, ya que la captura sobre el primer alimento vivo, puede considerarse como crítica. Asimismo, una de las ventajas de suministrar alimentos vivos como los microcrustáceos, estos están en constante movimientos y muy rara vez, llegan al fondo por cuanto no se descomponen afectando la calidad del agua.

Se concluyó que mediante la reproducción artificial aplicando el método seco, se obtuvo un alto porcentaje de ovas fecundadas y alevinos, resultando los microcrustáceos el alimento recomendado por ser natural y presentar todas las características morfo-fisiológicas para su consumo y desarrollo. 


\section{REFERENCIAS}

Argota, G. (2015). Aplicación gecotoxic para predicción de riesgo ambiental: caso estudio sobre mortandad de peces en la bahía interior del lago Titicaca-Puno, Perú. Campus, 20, 11-20.

Atencio, V. (2001). Producción de alevinos de especies nativas. Revista MVZ Córdoba, 6(1), 9-14.

Atencio, V., Kerguelen, E., Naar, E. \& Petro, R. (2013). Desempeño reproductivo del bocachico Prochilodus magdalenae inducido dos veces en un mismo año. Rev. MVZ Córdoba, 18(1), 33043310 .

Buschmann, A. H. (2001). Impacto ambiental de la acuicultura. El estado de la investigación en Chile y el Mundo. Santiago, Chile: Terram Publicaciones.

Centro de Investigación y Desarrollo Acuícola Boliviano: CIDAB. (2002). Desarrollar la capacidad de programas de pesca artesanal en Bolivia. Recuperado de http://www.altperubolivia.org/ Web Bio/PROYECTO/Docum bolivia/21.24\%20P1.pdf

Chura, C.R. \& Mollocondo, H.H. (2009). Desarrollo de la acuicultura en el Lago Titicaca (Perú). Aqua TIC, 31, 6-19.

Constantini, M.L., L. Savetta, G. Mancinelli \& L. Rossi. (2004). Spatial variability of the decomposition rate of Schoenoplectus tatora in a polluted area of Lake Titicaca. Journal of Tropical Eco$\log y, 20,325-335$.

Huet, M. (1988). Tratado de piscicultura ( $2^{a}$ ed.). Madrid, España: Ediciones Mundi-Prensa.
Llanos, C. \& Scotto, C. (2014). Comparación entre la obtención de crías por cruza natural versus la utilización de un inseminador artificial en el pez ornamental Xiphophorus helleri (Heckel, 1848) (Cyprinodontiformes: Poeciliidae). AquaTIC, 40, 21-31.

Lenis, G., Restrepo, L.F. \& Cruz, P.C. (2009). Evaluación de tres protocolos de tratamiento hormonal sobre el diámetro de ovocitos de sabaleta Brycon henni. Revista Colombiana de Ciencias Pecuarias, 22(2), 131-142.

Mantilla, B. (2004). Acuicultura: Cultivo de truchas en jaulas flotantes. Universidad Nacional del Altiplano. Lima, Perú: Editorial Palomino E.I.R.L.

Meneses, C.H. \& Flores, M.O. (1995). Reproducción de Especies Icticas Nativas de la Cuenca del Lago Titicaca por medio artificial en laboratorio. Proyecto Especial Binacional Lago Titicaca. Perú: INADE. Dirección de Recursos Hidrobiológicos.

Organización de las Naciones Unidas para la alimentación y la Agricultura (2002). Fisheries and Aquaculture Department. Recuperado de http:/www.fao.ogr/ fishery/culturedspecies/Oncorhynchus_mykiss

Samantray, P., Mishra, B.K., Panda, C.R. \& Rout, S.P. (2009). Assessment of water quality index in Mahanadi and Atharabanki rivers and Taldanda canal in Paradip Area, India. Journal of Human Ecology, 26, 153-161. 
